Addendum

\title{
Addendum: Cohen, M.H. OJ 287 as a Rotating Helix. Galaxies 2017, 5, 12
}

\author{
Marshall H. Cohen \\ Department of Astronomy, California Institute of Technology, Pasadena, 91125 CA, USA; mhc@astro.caltech.edu;
} Tel.: +1-626-395-4977

Received: 27 April 2018; Accepted: 22 May 2018; Published: 28 May 2018

check for updates

Abstract: In an earlier paper it was erroneously stated that the inner jet of OJ 287 has a right-hand helical structure. In this addendum we show that the observations underlying this statement are ambiguous, and that they cannot determine the rotation sense.

Keywords: BL Lacertae objects: individual (OJ 287); galaxies: jets; magnetohydrodynamics (MHD)

In Cohen [1] it was stated that the helical structure in the inner jet of OJ 287 has a right-hand twist. This conclusion was based on the evolution of the ridgelines of the object, but we now recognize that the observations are ambiguous. The penultimate sentence of the second paragraph of Section 3 ("The jet rotation ... Figures 2 and 3.") should be deleted. In addition, the third paragraph of Section 3 ("In Figures 2 and 3 ... right-hand helix.") should be deleted, and replaced by the following paragraph.

In Figures 2 and 3, the inner portions of the ridgelines rotate CW and in Figure 4 they lie in the sky plane, first north of the axis and then, from about 2000 to 2010, south of the axis. We assume that these motions are projections of rotations on a cone around the axis, of the long curves that represent the jet in Figure 4. These projections all appear to start in the $-x$ (west) direction, and Figure 4 shows the jet successively in black, red, and green at $\varphi=0^{\circ},-90^{\circ}$ and $-180^{\circ}$. This corresponds to a $\mathrm{CW}$ rotation on the cone, as seen by the observer. However, the deprojection could have been made the opposite way, with CCW rotation, with $\varphi=0^{\circ},+90^{\circ}$ and $180^{\circ}$, because the cone has a small intrinsic opening angle, $\alpha_{0}$. The apparent opening angle, $\alpha_{a p p}$, is measured in Figure 2 as the angle between the axis and the peak of the curves around 1997, and is about $22^{\circ}$. The relation connecting these angles is $\tan \alpha_{o}=\tan \alpha_{a p p} \sin \theta_{o}$; and, since $\alpha_{a p p}$ is clearly less than $45^{\circ}, \alpha_{o}<\theta_{o}$ and the entire cone is projected to the west. Both rotation senses are allowed by the observations.

\section{References}

1. Cohen, M.H. OJ 287 as a Rotating Helix. Galaxies 2017, 5, 12. [CrossRef]

(C) 2018 by the author. Licensee MDPI, Basel, Switzerland. This article is an open access article distributed under the terms and conditions of the Creative Commons Attribution (CC BY) license (http:/ / creativecommons.org/licenses/by/4.0/). 